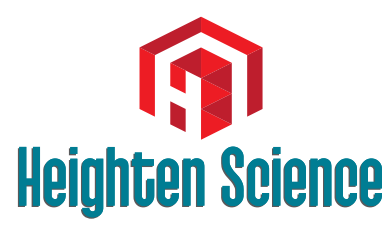

P U B L I C A T I O N S Corporation

\title{
Cystic adventitial disease of the external iliac artery with disabling claudication: A case report and short review
}

\author{
Nalaka Gunawansa* \\ Consultant Vascular, Endovascular and Transplant Surgeon, National Institute of Nephrology \\ and Transplant, Colombo, Sri Lanka
}

\begin{abstract}
*Address for Correspondence: Nalaka Gunawansa, Consultant Vascular, Endovascular and Transplant Surgeon, National Institute of Nephrology and Transplant, Colombo, Sri Lanka, Tel: + 94773 737644; Email: vascular@ drnalakagunawansa.com
\end{abstract}

Submitted: 03 May 2018

Approved: 02 July 2018

Published: 03 July 2018

Copyright: @2018 Gunawansa N. This is an open access article distributed under the Creative Commons Attribution License, which permits unrestricted use, distribution, and reproduction in any medium, provided the original work is properly cited

Keywords: Cystic adventitial disease; Claudication; Atherosclerosis; lliac artery

A) Check for updates

\section{Abstract}

Chronic occlusive arterial disease of the periphery is primarily caused by atherosclerotic disease. In young patients with no identifiable risk factors for atherosclerosis, who present with symptoms of claudication or critical ischemia, other rare causes need to be suspected. Cystic adventitial disease is one such condition affecting young healthy patients. Although it has been reported most commonly in relation to the popliteal artery, other sites including the iliac artery can also get affected. Isolated short segment stenosis or occlusion can lead to significant disabling symptoms restricted to one side. Imaging studies show pristine arterial anatomy with no evidence of systemic atherosclerotic disease and an isolated area of luminal stenosis. Definitive treatment involves open surgical excision with interposition grafting for optimal long-term results. We report a case of cystic adventitial disease affecting the external iliac artery in an otherwise healthy young man.

\section{Introduction}

The commonest aetiology of Chronic Occlusive Arterial Disease (COAD) is atherosclerosis. Buergers disease is less common, primarily affecting young male smokers. In younger patients with no risk factors for atherosclerosis or Buergers, other rare disease processes have been described. Cystic adventitial disease (CAD) is one such condition reported as a cause of isolated popliteal artery occlusion causing claudication and occasionally critical limb ischaemia (CLI) [1].

CAD of the peripheral arteries was first reported in 1947 by Atkins and colleagues [2]. Since then, under 400 cases have been reported in the literature with CAD as a cause of non-atheromatous occlusive arterial disease in the young. Among these, the great majority have been reported affecting the popliteal artery with only 35 reported cases affecting the ileo-femoral segment [3].

CAD affects isolated short segments of an artery with cystic myxoid degeneration arising from the adventitia. The cysts expand and encroach the arterial lumen, resulting in focal stenosis or occlusion in an otherwise pristine artery. The clinical picture is that of a healthy young patient having significant claudication of rapid onset and progression. The following report is of such a patient with CAD of the left external iliac artery (EIA).

\section{Case Report}

A 46-year-old male presented with disabling claudication in his left leg over a period of 2 months. His claudication distance was $30 \mathrm{~m}$ and was significantly hampered by the disability. There was no claudication in the right leg. He was a non-smoker and had no history of diabetes or hypertension. There was no family history suggestive of 
accelerated atherosclerosis or hyperlipidaemia. On examination, he had normal pulses on the right leg and both arms. On the left, the femoral, popliteal and distal pulses were all absent. The ankle brachial pressure index was 1 on the right 0.5 on the left side.

A computerized tomographic angiogram (CTA) showed isolated short segment severe stenosis in the left EIA with otherwise pristine arterial anatomy (Figure 1). Considering his young age, presentation and the imaging findings, a clinical diagnosis of CAD was made, and he was offered surgical revascularization. Surgery was performed under spinal anaesthesia. The EIA was exposed via a curvilinear incision along the left iliac fossa and medial retraction of the peritoneum. External appearance of the EIA was unremarkable with no connection to the hip joint. Proximal and distal control was achieved, and the occluded segment was opened with a vertical arteriotomy. This exposed a thick gelatinous substance originating from the adventitia extending in to the lumen. There were no atherosclerotic plaques and the adjacent intima was free of macroscopic disease. The affected segment (approximately $4 \mathrm{~cm}$ ) was excised and sent for histology (Figure 2). The EIA was reconstructed with an interposition graft, $7 \mathrm{~mm}$ expanded poly-tetra fluoroethylene (ePTFE). He was continued on aspirin postoperatively.

Post-operative recovery was uncomplicated, and the patient was discharged on day -04. Out-patient follow-up was also unremarkable, with complete recovery over the ensuing weeks. There were no residual symptoms. At 18 months post-operative, he remains fully active with no claudication and normal distal pulses on both legs. The histology of the resected segment of artery was confirmed as CAD (Figure 3).

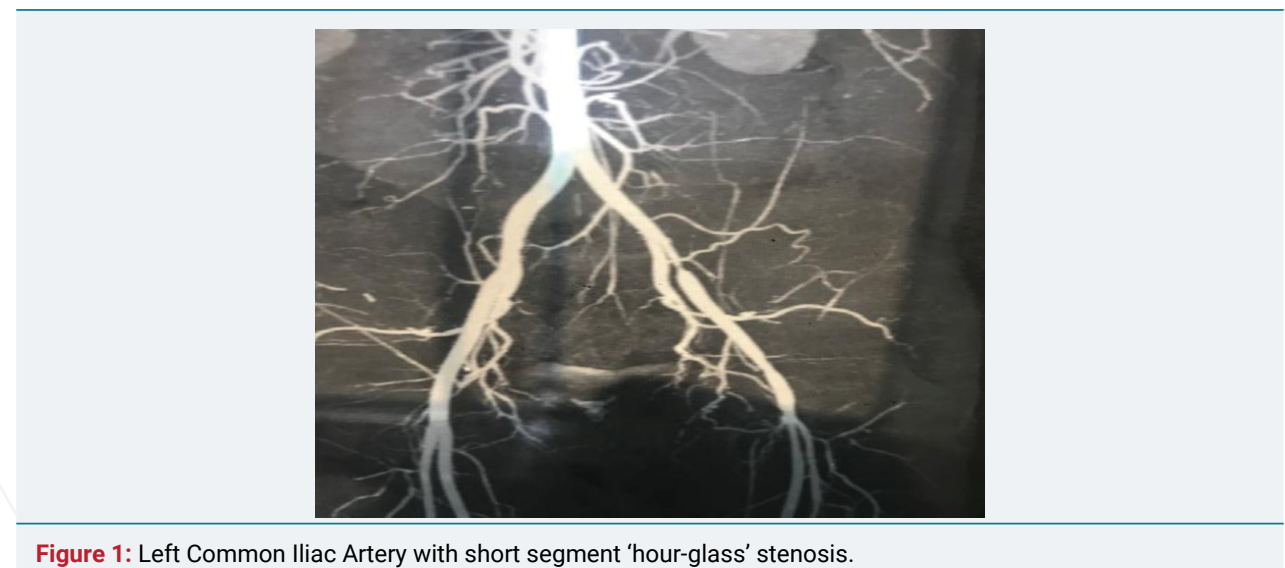

Figure 1: Left Common Iliac Artery with short segment 'hour-glass' stenosis.

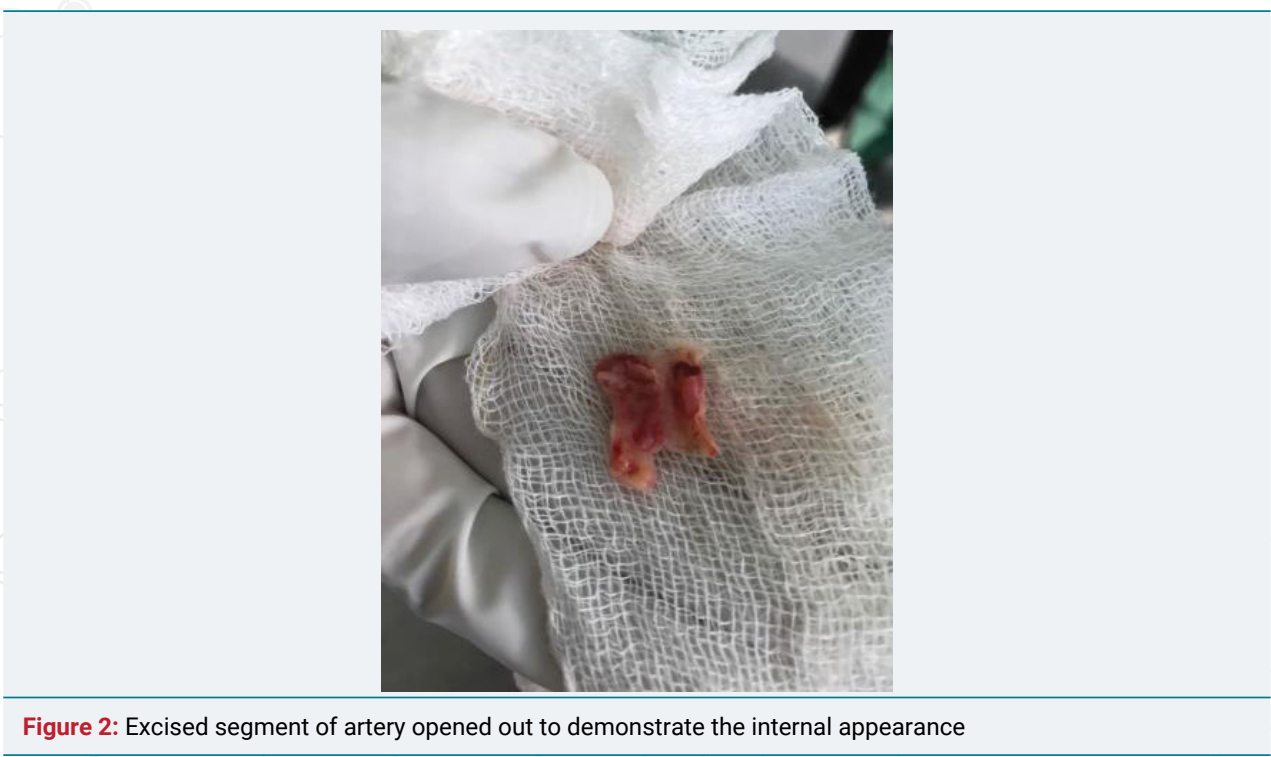




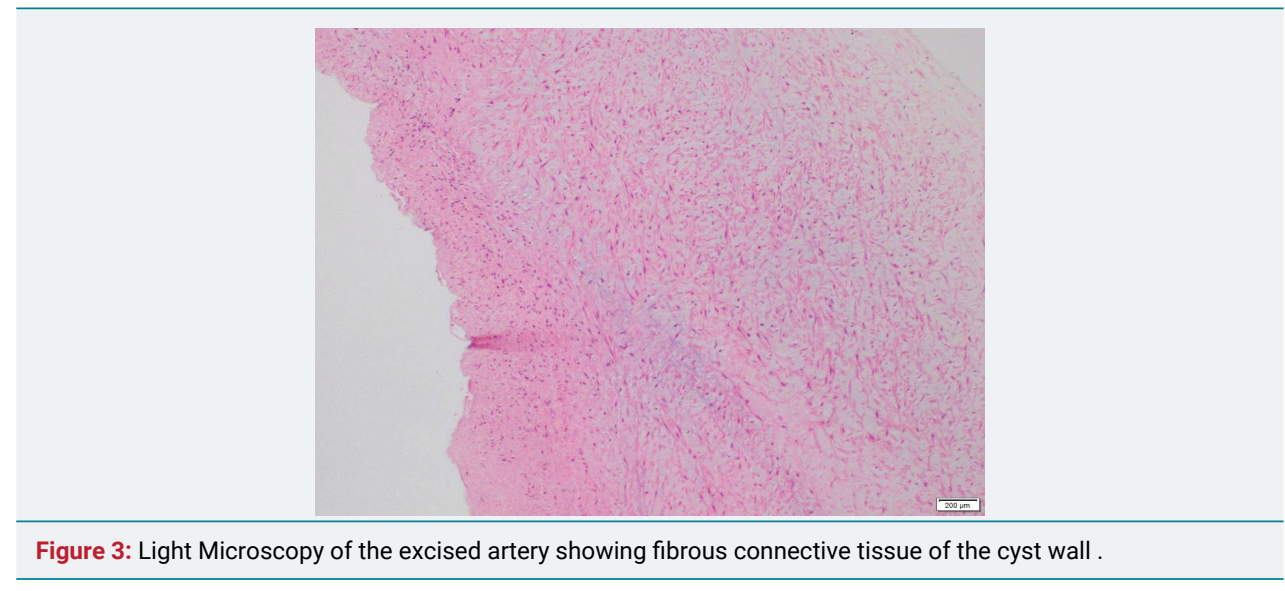

\section{Discussion}

CAD has been reported as an extremely rare cause of non-atheromatous arterial occlusion in younger patients [3]. The typical age of onset is the fifth decade as was the case in the index patient. There is a significant male preponderance, male to female incidence of 15:1 [4].

Since the first reported case in 1947, the great majority of cases reported have been involving the popliteal artery. The first reported case of CAD affecting the ileo-femoral segment came from Kuijpers and colleagues (1969) [5]. The largest series of CAD affecting the EIA has been reported by Ishikawa and colleagues in 1987 [6].

There are several hypotheses regarding the aetiology of CAD.

1. Mechanical trauma: Repetitive trauma to affected artery due to its close proximity with a major joint,

2. Embryologic: mucin-secreting mesenchymal cells from the joints are deposited in the adjacent arterial adventitia during development,

3. Direct extension: extension from the constituents of an adjacent joint (similar to a ganglion). This direct extension theory is currently the most accepted hypothesis, describing a ganglionic synovial cyst herniating and tracking along the arterial supply to the joint, back to the main artery.

4. Connective tissue theory: an additional theory states that CAD is part of a more generalized connective tissue disorder.

Progressive enlargement of the cysts leads to significant luminal narrowing and distal ischaemia. The symptoms usually have a short duration and rapid progression. Clinical examination reveals absent or diminished pulses in one limb, often indistinguishable from atherosclerotic disease. ABPI assessment shows significant reduction in the affected limb as seen in the index patient.

Duplex scan can be used to visualize the area of stenosis with remarkable accuracy, especially in the popliteal artery. However, in supra-inguinal disease, CTA or Magnetic resonance angiography (MRA) becomes more accurate in diagnosing the lesion. Concentric disease gives rise to a unique "hour- glass" narrowing of the artery as was seen in the index patient (Figure 1). Conversely, the eccentric variety affecting one side of the vessel results in a curvilinear luminal narrowing. The absence of generalized atherosclerotic disease and significant collaterals along with isolated short segment stenosis close to a major joint are all pointers towards a preliminary diagnosis of CAD.

Endovascular interventions including guided aspiration have been reported with limited success. The high viscosity of contents and multi-locular nature often 
makes it impossible to perform complete aspiration [4]. Furthermore, the chief drawback of aspiration is the high recurrence rate due to residual mucin-secreting cells. Percutaneous angioplasty and stenting has been attempted with limited success also due to high rates of recurrence, sometimes reported as early as 24 hours after the procedure. Furthermore, angioplasty carries the additional risk of damaging an otherwise healthy unaffected intima thereby increasing the potential for thrombosis while deployment of stents near a major joint in a young active patient becomes prohibitive [3].

Some have described local enucleation or circumferential excision without breaching the intima, with a reported recurrence rate around 10\% [7]. However, with near-total occlusion rather than narrowing, as seen in our index patient, the best recommended approach remains excision with interposition graft.

\section{Conclusion}

- In young, otherwise healthy patients who present with unilateral claudication without identifiable risk factors for atherosclerosis, CAD should always be entertained as a differential diagnosis.

- Contrast imaging will show pristine arterial anatomy with isolated short segments of stenosis or occlusion.

- Definitive diagnosis is by intra-operative findings and histological confirmation of excised arterial segment

- Complete surgical resection and interposition grafting offers the best long-term outcome for these young patients who are often in the peak of their productive life.

Explicit written consent was obtained from the patient to share the relevant clinical details and images for educational purposes.

\section{References}

1. Hall RI, Proud G, Chamberlain J, McNeil IF. Cystic adventitial disease of the common femoral and popliteal arteries. Br J Surg. 1985; 72: 756-758. Ref.: https://tinyurl.com/y9bkpyqn

2. Atkins HJ, Key JA. A case of myxomatous tumour arising in the adventitia of the left external iliac artery. Br J Surg. 1947; 34: 426-427. Ref.: https://tinyurl.com/y7qmubg3

3. Baxter AR, Garg K, Lamparello PJ, Mussa FF, Cayne NS, et al. Cystic adventitial disease of the popliteal artery: Is there a consensus in management? Vascular. 2011; 19: 163-166. Ref.: https://tinyurl.com/ybojkjrr

4. Paravastu SC, Regi JM, Turner DR, Gaines PA. A contemporary review of cystic adventitial disease. Vasc Endovascular Surg. 2012; 46: 5-14. Ref.: https://tinyurl.com/y7lyh7dh

5. Kuijpers $P$, Mol P, Hoefsloc F. Idiopathic cystic degeneration of the left common femoral artery. Semantic Scholar. Arch Chir Neerl. 1969; 21: 77-84. Ref.: https://tinyurl.com/ychcljzg

6. Ishikawa K. Cystic adventitial disease of the popliteal artery and of other stem vessels in the extremities. Jpn J Surg. 1987; 17: 221-229. Ref.: https://tinyurl.com/ybq3j5zy

7. Stierli P, Mauch J, Koella C, Huber A, Eugster T, et al. Circumferential removal of the adventitia for cystic degeneration of the popliteal artery. Br J Surg. 2005; 92: 56-57. Ref.: https://tinyurl.com/y8qoo34q 